\title{
Simulating the Dynamics of Auroral Phenomena
}

\author{
GLADIMIR V. G. BARANOSKI and JUSTIN WAN \\ University of Waterloo \\ JON G. ROKNE \\ University of Calgary \\ and \\ IAN BELL \\ University of Waterloo
}

\begin{abstract}
Simulating natural phenomena has always been a focal point for computer graphics research. Its importance goes beyond the production of appealing presentations, since research in this area can contribute to the scientific understanding of complex natural processes. The natural phenomena, known as the Aurora Borealis and Aurora Australis, are geomagnetic phenomena of impressive visual characteristics and remarkable scientific interest. Aurorae present a complex behavior that arises from interactions between plasma (hot, ionized gases composed of ions, electrons, and neutral atoms) and Earth's electromagnetic fields. Previous work on the visual simulation of auroral phenomena havefocused on static physical models of their shape, model ed from primitives, likesine waves. In this article, we focus on the dynamic behavior of the aurora, and we present a physically-based model to perform 3D visual simulations. The model takes into account the physical parameters and processes directly associated with plasma flow, and can be extended to simulate the dynamics of other plasma phenomena as well as astrophysical phenomena. The partial differential equations associated with these processes are solved using a complete multigrid implementation of the electromagnetic interactions, leading to a simulation of the shape and motion of the auroral displays. In order to illustrate the applicability of our model, we provide simulation sequences rendered using a distributed forward mapping approach.
\end{abstract}

Categories and Subject Descriptors: I.3.7 [Computer Graphics]: Three-Dimension Graphics and Realism

General Terms: Algorithms

Additional Key Words and Phrases: Atmospheric effects, natural phenomena, plasma phenomena, rendering

\section{INTRODUCTION}

The Aurora Borealis, or "Northern Lights", and Aurora Australis, its southern counterpart, are light emissions caused by the collision of an ionized stream of charged particles with high altitude atmospheric atoms and molecules [Eather 1980]. These emissions are considered to be among the most fascinating and mysterious of Nature's spectacles by those who have been fortunate enough to see them. Taylor in National Geographic calls them "Earth's Grand Show of Lights" [Taylor 2001]. An inherent characteristic of auroral displays is that they move and can change shape both slowly and

Funding is gratefully acknowledged from both the National Sciences and E ngineering Council of Canada (NSE RC Grants 213281 , 239162, and 691266) and the Canada Foundation for Innovation (CFI Project 6218).

Author's address: G. V. G. Baranoski, Natural Phenomena Simulation Group, School of Computer Science, University of Waterloo, 200 University Avenue West, Waterloo, ON N2L 3G1, Canada.

Permission to make digital or hard copies of part or all of this work for personal or classroom use is granted without fee provided that copies are not made or distributed for profit or direct commercial advantage and that copies show this notice on the first page or initial screen of a display along with the full citation. Copyrights for components of this work owned by others than ACM must be honored. Abstracting with credit is permitted. To copy otherwise, to republish, to post on servers, to redistribute to lists, or to use any component of this work in other works requires prior specific permission and/or a fee. Permissions may be requested from Publications Dept., ACM, Inc., 1515 Broadway, New York, NY 10036 USA, fax: +1 (212) 869-0481, or permissions@acm.org. (C) 2005 ACM $0730-0301 / 05 / 0100-0037 \$ 5.00$ 
rapidly. These complex and stochastic motions are directly related to the plasma processes. In this article, a novel physically-based model to perform 3D visual simulations of the dynamics of the auroral phenomena is presented. Furthermore, auroral surges (Figure 14), which have not been previously simulated, are also rendered. This model, therefore, enables the simulation of shape and motion of the aurorae in a realistic manner.

The aurorae are frequently visible at high latitudes. They can also be seen less frequently at lower latitudes, especially around solar sunspot maxima (a period of great solar turbulence that occurs approximately every 11 years [Odenwald 2000]). For instance, the last solar cycle of the 20th century reached its maximum in the year 2000, and it produced amazingly bright aurorae for the next couple of years. Aurorae are also a prominent visual phenomena in space as seen from spacecrafts orbiting the Earth. Further afar, they occur on planets with strong magnetic fields such as J upiter and Saturn. Hence, the simulation of auroral displays is also of interest for the production of night sky visualization sequences used in planetarium shows. Moreover, the al gorithms used in our plasma-model may be applied to the simulation of other plasma-related phenomena, such as gas discharges encountered in neon and fluorescent lights, welding arcs, and lightning.

On the scientific side, the importance of the visual simulation of auroral dynamics is related to the links to plasma physics. As much as $99 \%$ of the matter in the universe is thought to exist in a plasma state [Bittencourt 1986; Chen 1984; NRC 1995]. The auroral displays provide a natural laboratory in which the complex workings of the plasma universe can be studied. Therefore, our simulations can be used in the evaluation of auroral theories as well as in the visualization of other plasma phenomena. Furthermore, plasma kinetic theory can be used to study the devel opment of galaxies, since, although stars in a galaxy are not charged, they behave like particles in a plasma [Bittencourt 1986; Chen 1984; NRC 1995]. Another area of application might be the study of the effects of nuclear explosions in the upper atmosphere. These explosions were found to create aurora-like displays [Dupont 2004].

Yet the main motivation for this research is the spellbinding majesty of a moving auroral display [Taylor 2001].

\subsection{Related Work}

The spectacular auroral motions and shape changes involve to some extent shear, meaning differential motions within different parts of a fluid or gaseous material. These motions are common in nature.

Shear eddies occur, for instance, on the surface of water in streams and in smoke curling up from a fire, and they are responsible for clear-air turbulence and gusty winds [Davis 1992]. The shear found in the aurorae is associated with plasma processes, and the most fascinating consequences are the formation of rotational distortions having different spatio-temporal scales.

Blinn and Wolf [Upson et al. 1987] produced a film in 1983 entitled "J upiter's Magnetosphere: The Movie". In this film, representations of spacecraft observations as well as analytic models of the plasma and magnetic fields in the J ovian system were employed to visualize the morphology and dynamical structure of J upiter's magnetosphere. To the best of our knowledge, this film represents the first use of a plasma model by the graphics community. Recent works involving plasma models in the graphics literature focus on the visual ization of plasma phenomena occurring in laboratory experiments. For example, Parker et al. [1995] devel oped a gyrokinetic particle simulation to visualize a plasma turbulence in a Tokomak (an apparatus for producing controlled fusion reaction in hot plasma), and Ljung et al. [2000] presented a system for the animation of the evolution of an instability that gives rise to a plasma surfatron, a mechanism for particle acceleration of importance in particle accelerators, in the context of electron acceleration in astrophysical shocks and in the solar corona. It is relevant to note the work of Schussman et al. [2000] who presented techniques for the visualization of magnetic field data from the DIII-D Tokomak, a fusion facility that conducts both experimental and computational plasma research. 
Recently, Baranoski et al. [2000, 2003] presented a static physically-based al gorithm for the rendering of auroral displays which accounts for important auroral visual features such as their characteristic spectral and intensity variation. Their work, however, does not takeinto account the el ectromagnetic instabilities responsible for the timevarying behavior of the aurorae. Nonetheless, it provides an accurate framework for the rendering of auroral images. In this article, the dynamical behavior of the aurorae is modeled by taking into account physical parameters and processes directly associated with plasma flow. The set of partial differential equations (PDEs) associated with these processes is solved using a complete multigrid implementation of the electromagnetic interactions between charged particles in the ionosphere, enabling us to produce a visual display of the "dancing lights".

The simulation of natural phenomena involving shear and fluid flow is an active area of research in computer graphics [Foster and Metaxas 1997; Foster and Fedkiw 2001; Kajiya and Herzen 1984; Myazaki et al. 2001; Stam and Fiume 1993; Stam 1999]. A comprehensive review of computer graphics simulations of fluid flow is beyond the scope of this article; however, we should remark that the plasma instability responsible for the stochastic and complex nature of the aurorae is similar to the Kelvin-Helmholtz instability (KHI) for sheared fluids [Tajima 1989]. In spite of that, we believe that a more natural way to model plasma phenomena is to simulate the equations of plasma flow directly. The use of a plasma model allows the control of the simulations by auroral physically meaningful parameters. Moreover, the use of a plasma model enables the accurate reproduction of the rapid intermediate scale motions often seen in the aurorae such as the motion of rays and folds along auroral forms.

Recent works on night sky phenomena have focused on obtaining images that are not only realistic, but are also predictive. This objective is achieved by adhering to physical principles and incorporating measured data to the simulations. After all, it is far easier to control a simulation using familiar physical concepts than through arcane parameters as appropriately stated by Arvo [1995]. As illustrative examples of this trend, besides the work by Baranoski et al. [2000, 2003] mentioned above, we should note the works by Nadeau et al. [2001] and J ensen et al. [2001].

Nadeau et al. [2001] presented star and nebula visualization techniques for creating a 3D volumetric representation of the Orion Nebula, which was used in a planetarium daily show. J ensen et al. [2001] developed a physically-based model of the night sky which also has educational applications. Although the J ensen et al. model does not include auroral displays, their night sky images can be combined with our auroral simulations. They also highlighted the role of naturally illuminated night scenes in the history of image-making and their importance in the area of realistic rendering.

Simulating plasma instabilities as well as fluid instabilities involves the solution of systems of PDEs. In these situations, the production of simulation sequences used to depict time dependencies or to analyze motion requires that such systems be solved repeatedly. Usually standard numerical methods, such as the Conjugate Gradient (CG) and Fast Fourier Transform (FFT)[Golub and Loan 1989], are applied. Stam [1995, 1999] has highlighted the theoretical advantages of multigrid methods [Hackbusch 1985] for solving such systems. In order to solve the PDEs arising from our plasma simulations, we present a practical and fast multigrid implementation. We note that our multigrid implementation is matrix-free, that is, it does not store the coarse grid matrices. Previous multigrid implementations found in the computer graphics literature, such as the one by Witkin and Kass [1991] in the their texture synthesis algorithm, used coarse grids to speed up the computations of explicit steady state solutions. Here, we use multigrid in the context of time dependent problems, and in each implicit time step, the multigrid is applied to solve a linear system. It also worth noting that Weimer and Warren [1999] used a full multigrid approach for fluid flow which is appropriate for solving nonlinear equations. In our implementation, we used a V-cycle multigrid which is more appropriate for solving linear equations such as those arising in our simulations. 


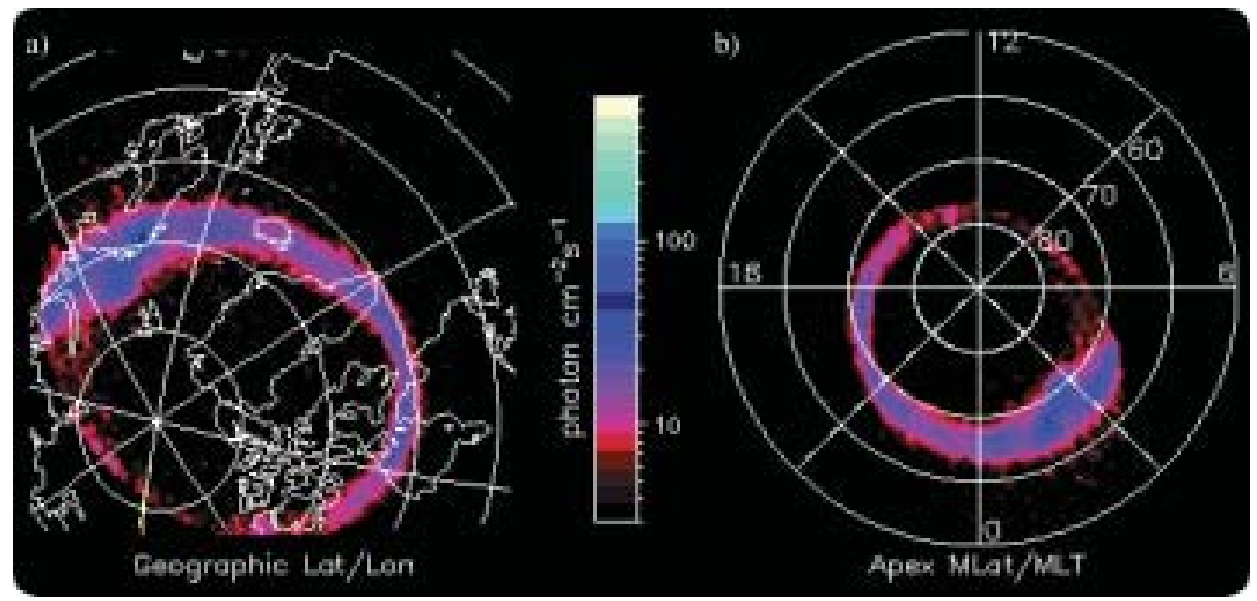

Fig. 1. Satellite snapshot of an auroral oval around the Earth's north magnetic pole. a) Viewed from the satellite position and b) viewed from a position normal to the Earth's north pole. (Courtesy of NASA Polar UItraviolet Imager.)

\subsection{Organization}

The next section provides an overview of relevant auroral physics concepts and describes the main motions and shape changes of the aurorae. In Section 3, the modeling framework used in this research is outlined. Section 4 presents the al gorithms used to simulate the dynamics of theauroral phenomena. The rendering issues are addressed in Section 5. Section 6 presents the results of our simulations and discusses performance issues. Section 7 outlines applications and directions for future work. Finally, Section 8 closes the article.

\section{AURORAL PHENOMENA}

In this section, we outlinethe main physical aspects directly related to the simulation of auroral dynamics. A reader interested in a detailed description of auroral physics is referred to more comprehensive texts on these phenomena [Brekke and Egeland 1994; Davis 1992; Eather 1980; J ones 1974; Omholt 1971]. The diagrams of auroral distortions presented in this section use as referencean auroral local coordinate system represented by xyz, where the z-axis corresponds to the direction parallel to the Earth's magnetic field vector $\vec{B}$, in the upwards direction, but not necessarily perpendicular to the ground.

\subsection{Overview}

The particles responsible for the col orful auroral displays are electrons that originate from solar flares and coronal mass ejections and become the "solar wind" [Burtnyk 2000]. After interacting with the Earth's magnetosphere, these particles migrate along the magnetic field lines, and "dive" towards oval regions around theE arth's magnetic pol es under certain conditions. These regions are called the auroral ovals. Figure 1 shows a satellite snapshot of an aurora oval around the Earth's north magnetic pole.

The shape of an aurora is determined by the energy and density of the electrons entering the atmosphere as well as the local variations in the Earth's magnetic and electric fields. The most common aurorae can be thought of as "curtains" of light emissions from "sheets" of falling el ectrons, which move al ong the Earth's magnetic field.

As the electrons travel down along the Earth's magnetic field lines, they suffer many random deflections which are caused by collisions with atoms of atmospheric gases such as oxygen and nitrogen. These deflections spread the electrons out horizontally. When the electrons collide with atmospheric 


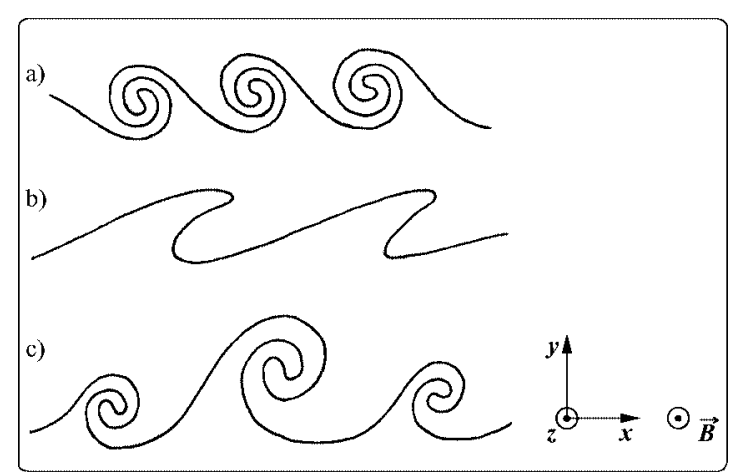

Fig. 2. Line drawings of cross sections of auroral arcs (not to scale) illustrating the three major shear-type rotational distortions: a) curls, b) folds, and c) spirals. The symbol $\odot$ denotes a vector normal to the plane of the paper.

constituents, the atoms are excited, and after a period of time, they may emit a photon. Statistically, several collisions must occur before a photon is emitted. As a result of these photon emissions, the curtains may be col ored, bright yellow-green at the bottom, perhaps red at the top, and a yellow-orange transition may also be present. Often they form arcs that can extend over $2000 \mathrm{~km}$ whose width (thickness) may vary from several hundred meters to several kilometers. These arcs can bend and fold, al most like real curtains, thereby generating bright vertical streaks.

\subsection{Auroral Shear-Type Distortions}

The sheets of precipitating electrons form an auroral stream that is subject to instabilities responsible for the shear-type rotational distortions seen in auroral arcs. Hallinan and Davis [1970] identified the three distinct types of distortions: curls, folds and spirals (Figure 2). Curls are small scale and have a diameter of $1 \mathrm{~km}$ or less. Folds are intermediate scale distortions and have a diameter of approximately $20 \mathrm{~km}$ or less. Spirals, or surges, are the largest auroral distortions and diameters are typically around 20-1300km [Partamies et al. 2001]. In addition to the different spatial scales, these phenomena have different temporal scales. Curls have a lifetime of a fraction of a second, while folds can exist for more than a second, and spirals may last minutes.

Curls and folds are responsible for distinct visual features present in auroral displays. The light emanating from convoluted folds in auroral displays often creates the impression of vertical, or nearvertical, "stripes" [Bryant 1999]. Despite their highly transient nature, curls are largely responsible for another important auroral feature, namely, electron beams evolving into thin field-aligned filaments or "rays". An auroral ray typically has a vertical dimension up to several hundred kilometers. Curls are usually $2-10 \mathrm{~km}$ apart, while folds are approximately $20 \mathrm{~km}$ apart. Spirals are also relatively common auroral structures, visible in more than $30 \%$ of the Defense Meteorological Satellite Program observations $^{1}$ [Davis and Hallinan 1976]. They can form a street of two or more spirals approximately $100 \mathrm{~km}$ apart. The spirals in the street usually have similar configuration and size, but they may also grow monotonically along the auroral display [Davis and Hallinan 1976].

\subsection{Auroral Motions}

As pointed out by Davis [1992], auroral motions and shape changes seem bewildering, yet they present some order when examined in detail. Many of the motions and shape changes associated with the auroral distortions are caused by warping of the incoming particle streams by the attendant magnetic

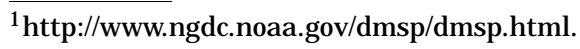




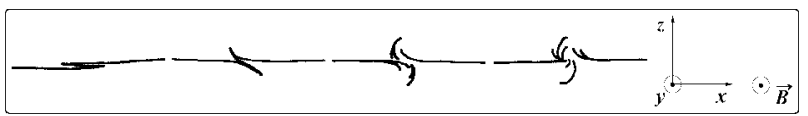

Fig. 3. Line drawings illustrating an auroral arc break-up. The symbol $\odot$ denotes a vector normal to the plane of the paper.

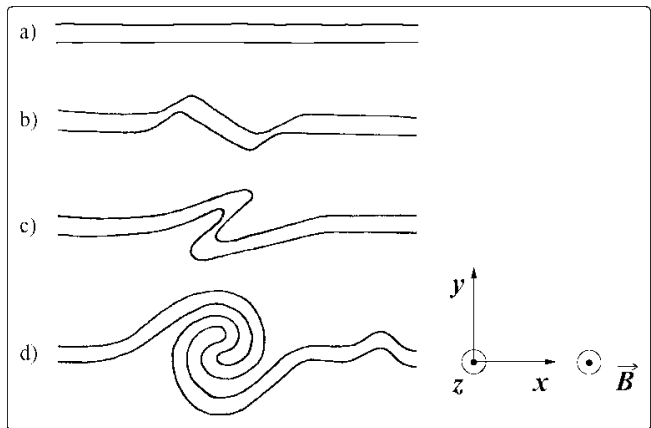

Fig. 4. Sketch showing different stages of an auroral warping process: a) initial arc, b) fold, c) further distorted fold, and d) spiral array. The symbol $\odot$ denotes a vector normal to the plane of the paper.

and electric fields. In this section, we outline the most important of these changes according to their spatial scale.

2.3.1 Small Scale. When an electron particle stream responsible for producing a small scale structure becomes slightly more dense in a region, it is likely to become contorted and devel op a curl [Davis 1992]. The curl formation process is similar to the spiral formation process and leads to essentially the same final configuration. However, the entire curl formation can occur so rapidly $(0.25-0.75$ s [Trondsen 1998]) that an observer on the ground has difficulty following the process.

2.3.2 Intermediate Scale. Once a curl vortex develops, it tends to cause the formation of another curl a short distance along the arc. The net result is the development of an array of evenly spaced curls along the arc, manifesting as rays. If another arc is nearby, the rays come under the influence of its electrical field and, therefore, move along the arc [Davis 1992]. If the process that leads to curl formation continues a bit longer, then small, uniformly spaced folds occur, and sometimes the arc may split into two separate arcs as the folds break apart (Figure 3).

Folds have apparent horizontal velocities in the range of $0-5 \mathrm{~km} / \mathrm{s}$ [Hallinan and Davis 1970]. The apparent horizontal velocities of curls lie in the range of $0-90 \mathrm{~km} / \mathrm{s}$, with preferred speeds in the range of $0-8 \mathrm{~km} / \mathrm{s}$ [Trondsen 1998]. To have a better idea of the magnitude of these speeds, recall that an auroral arc may extend over the entire field-of-view of an observer on the ground. For this observer the auroral rays seem to "travel" back and forth between two extreme points in the horizon in-few seconds.

2.3.3 Large Scale. When an incoming stream of electrons that produces an auroral arc develops a slight irregularity, the irregularity can grow [Hallinan 1976, 1981; Levy and Hockney 1968; Partamies et al. 2001]. As it does, the stream becomes distorted, carrying incoming particles to new locations in the auroral domain [Davis 1992]. The growth of this irregularity depends on the thickness of the el ectron stream. The larger the stream length-to-thickness ratio, the faster the irregularity grows with the distance [Hallinan 1976; Levy and Hockney 1968]. Figure 4 shows an auroral arc being affected by 
the warping process, first developing a fold, and then progressing into a complex spiral shape. These large auroral vortices have an average speed of $4 \mathrm{~km} / \mathrm{s}$ [Partamies et al. 2001].

\section{MODELING FRAMEWORK OVERVIEW}

Computer models of plasma can be broadly divided in three kinds: Eulerian, Lagrangian and hybrids of these [Hockney 1966]. The Eulerian model assumes that plasma behaves like a fluid, or that it obeys macroscopic differential equations for average quantities. The Lagrangian model follows the motion of a large number of particles as they move in time under their mutual electromagnetic interactions. Although this model acts on the microscopic particle level, it allows the particle ensemble to "make its own mind" about the macroscopic and collective behavior. This aspect is relevant in the simulation of phenomena with noticeable stochastic traits and different spatio-temporal scales such as aurorae. We use a hybrid Eulerian-Lagrangian approach in our modeling of auroral phenomena. Such a hybrid approach has been successfully used in computational physics simulations for many years [Hockney 1966; Levy and Hockney 1968; Hockney and Eastwood 1988].

In many scientific fields, the behavior of natural phenomena is described using PDEs. Recently, PDE-based modeling and numerical techniques from computational fluid dynamics (CFD) have been applied to computer graphics, especially for animations involving fluids. For instance, in Fedkiw et al. [2001], smokewas modeled by inviscid Euler equations. Level set and semi-Lagrangian techniques were applied in the numerical computation. In Rasmussen et al. [2003], large scale smoke phenomena were model ed by 2D incompressible Euler equations, together with the Kolmogorov spectrum. In Kunimatsu et al. [2001], water was modeled by Navier-Stokes equations. The Eulerian approach was adopted and the volume-of-fluid method was used to track free water surfaces. Another approach is to use semiLagrangian and level set method with inertialess particles [Foster and Fedkiw 2001]. The particle level set technique was further modified in Enright et al. [2002] to obtain more accurate results. In Nguyen et al. [2002] and Feldman et al. [2003], fireand expl osion were model ed by incompressibleEuler equations. The latter used nonzero velocity divergence to capture fluid expansion during explosion. Cloud simulation was modeled by Boussinesq's equations in Miyazaki et al. [2002].

At first glance, the similarities between neutral fluids and plasma [Tang 2002] might suggest that the CFD formulation would be sufficient to model the behavior of both media. However, in order to perform predictive simulations of the dynamics of plasma phenomena, one must account for their distinctive features. For example, turbulence dynamics in plasmas invol ve el ectromagnetic fiel ds and a much larger number of relevant variables than for neutral fluids [Yoshizawa et al. 2001]. Furthermore, while the effects of gl obal boundary conditions, such as wall boundaries, often play an important rolein the growth of neutral fluid disturbances [Drazin and Reid 1981; Hinze 1975; Yoshizawa 1998], plasma turbulence is greatly affected by spatial inhomogeneities and plasma configurations. These inhomogeneities are coupled together to drive or suppress turbulent fluctuations, which often have a very long correlation length along the magnetic field line and are quasi-two-dimensional [Yoshizawa et al. 2001]. Hence, to predictively simulate structure formation and transitions in plasma phenomena, such as the aurorae, one needs to account for the mutual interactions between plasma inhomogeneities and the electric-field structure and fluctuations.

Although the physical laws that affect the behavior of plasma phenomena, such as the Maxwell's equations, are well known, progress in this area was only made possible by advances in computational methods [Gombosi et al. 2004; Tang 2002]. Generally, an analytical, closed-form solution for these equations is difficult to obtain. One can only obtain a unique solution by imposing various restrictions [Volakis and Kempel 1995], and they are normally solved using numerical methods such as finite difference (FD) [Taflove 2000], finite volume (FV) [Anderson 2001], and finite element (FE) [Silvester and Ferrari 1990] methods and hybrids of these [Volakis and Kempel 1995]. Among these methods, 
FD's are the most commonly applied to the PDE formulation of Maxwell's equations in the time domain [Anderson 2001]. This method is based on the straight discretization of the differential equations, and it is suitable for modeling complex media [Kärkkäinen 2002]. This motivated its use in the simulation al gorithms described in this article. In order to accel er atetheconvergence of theiterativesol utions of the PDEs, we used a multigrid method. Recently, Aruliah and Asher [2002] al so used a multigrid scheme to speed-up the simulation of three-dimensional el ectromagnetic problems in geophysical regimes. While in our simulations multigrid is used to solve the Maxwell's potential equation directly, Aruliah and Asher [2002] used a multigrid scheme to approximately invert a block diagonal matrix, which, in turn, was used as a preconditioner for the discretization matrix.

The "electrons" in a plasma simulation correspond to millions of physical electrons. Provided that the physical phenomena have wavelengths that are long, compared with the average electrons spacings, and time scales that are short, compared with the time for the graininess to have significant effect, this description of "electrons" gives an accurate representation and leads to the concept of computational "superparticle" [Hockney and Eastwood 1988]. The graininess introduced by the paucity of superparticles is minimized by smoothing the short-range forces. One way of regarding the superparticles is as finite-sized clouds of electrons, where the position of a superparticle is the center of mass of the clouds and their velocities the mean velocities of the clouds [Hockney and Eastwood 1988].

We used a similar representation in our simulations in order to minimize computational overhead. Instead of tracking the around $10^{12}$ individual plasma particles that cause auroral phenomena, we follow the path of superparticles which we regard as beams of electrons. As such, we use normalized values for the physical quantities involved in our simulations, namely, charge, mass, and permissivity. The Lagrangian treatment is used to account for the interactions among beams and between beams and atmospheric constituents. The Eulerian treatment is applied when spatial scales much greater than the relatively small amplitude motions of the beams are considered.

The usual approach in computer experiments is to devise a model which is sufficiently detailed to reproduce the important physical aspects faithfully and yet not so detailed that the calculations become impractical. This approach becomes even more pertinent when the main purpose is to produce visual simulations whose purpose is to highlight the qualitative features of a given phenomenon. Selecting the "best" modeling approach depends on the relevant physical length and time scales.

The motion of the electron beams has two components: parallel and perpendicular to the electron stream. The parallel component is associated with the kinetic energy of the incoming particles. The perpendicular component is duetothewarping of the el ectron stream. The differences of themagnitudes of the time scales of these two components [Hallinan 1981; Brekke and Egeland 1994] means that they can be decoupled without incurring a loss of realism in their visual three dimensional representation.

The key stage of our modeling framework is the simulation of the warping process, which changes the perpendicular velocity, $\overrightarrow{\mathrm{V}}^{\perp}$, and the position of electron beams, P, at the top of the electron stream, at each instance of time. The mathematics of this process can be described by an electro-magnetic version of the Kelvin-Helmholtz instability (KHI) [Hallinan 1976, 1981; Levy and Hockney 1968; Partamies et al. 2001]. Section 4 describes the warping algorithm used in our simulations in detail.

The position of each beam at the top of the electron stream is considered as the starting point of its descending trajectory. The position update resulting from the warping process is associated with a steady state of incoming particles. A precipitating electron beam is tracked downwards until it becomes indistinguishable from the ambient electron population. During their descending trajectories, the electron beams may be deflected several times due to collisions with atmospheric constituents. Light emissions may occur at these deflection points, causing the auroral displays. These emissions are mapped to the screen plane of a virtual camera using a splatting approach [Westover 1991]. Rendering issues are further examined in Section 5. 
The auroral dynamics simulation uses the auroral local coordinate system defined in Section 2 . The mapping of auroral emissions for rendering purposes uses a global coordinate system represented by $\mathrm{XYZ}$, where the $\mathrm{Y}$-axis corresponds to the direction normal to the ground.

\section{DYNAMICS SIMULATION}

The electron beams interact with one another through the electric field, $\vec{E}$, generated by their mutual electrostatic repulsion, and the Earth's magnetic field, $\vec{B}$. Thus, the Lorentz force on an electron beam $e_{k}$ with charge $q_{k}$, moving with a perpendicular velocity $\vec{v}_{k}^{+}$is

$$
\overrightarrow{\mathrm{F}}_{\mathrm{k}}=\mathrm{q}_{\mathrm{k}}\left(\overrightarrow{\mathrm{E}}+\overrightarrow{\mathrm{v}}_{\mathrm{k}}^{\perp} \times \overrightarrow{\mathrm{B}}\right),
$$

and the motion of an electron beam of mass $m_{k}$ is determined by Newton's law

$$
m_{k} \frac{d \vec{v}_{k}^{\perp}}{d t}=\vec{F}_{k} \text {. }
$$

Thus, to update the positions of the electron beams, we need to compute $\vec{E}$ and $\vec{B}$. In the auroral warping simulation, the Earth's magnetic field is modeled as a uniform magnetic field of strength $|\vec{B}|$. At any instance of time, the electron beams and, hence $\vec{E}$, are located in the plane perpendicular to $\vec{B}$. By Gauss' law, $\vec{E}$ satisfies

$$
\nabla \cdot \vec{E}=\frac{\rho}{\epsilon_{0}},
$$

where $\epsilon_{0}$ is the permissivity of free space, and $\rho$ is the charge density function

$$
\rho(\mathrm{P})=\sum_{\mathrm{k}=1}^{\mathrm{N}} \mathrm{q}_{\mathrm{k}} \delta\left(\mathrm{P}-\mathrm{P}_{\mathrm{k}}\right) .
$$

Here we model the small mass, low density el ectron beams with charge $q_{k}$, at the position $P_{k}$, as point particles via the Dirac delta function $\delta(\cdot)$. From the Maxwell equations, which describe the dynamics of charged particles interacting with an electromagnetic field, we obtain

$$
\nabla \times \vec{E}=0 .
$$

The el ectrostatic potential $\phi$ is introduced such that

$$
\overrightarrow{\mathrm{E}}=-\nabla \phi,
$$

and the divergence of the potential gradient results in the Poisson Equation given by

$$
-\nabla^{2} \phi=\frac{\rho}{\epsilon_{0}}
$$

where $\nabla^{2} \phi$ is the Laplacian of the potential. By solving Equations (2) and (7) over time, we can model the perpendicular motion of the electron beams, which is the crucial aspect of the auroral dynamics.

\subsection{Warping Algorithm}

The potential Equation (7) and the electron beam velocity Equations (2) form a coupled continuousparticle system, which is simulated using the particle-mesh method exploiting the force-at-a-point formulation and the field equation for the potential. For the velocity equation, we use a Lagrangian grid approach, whereas for the potential equation, we use an Eulerian approach as suggested by Levy 


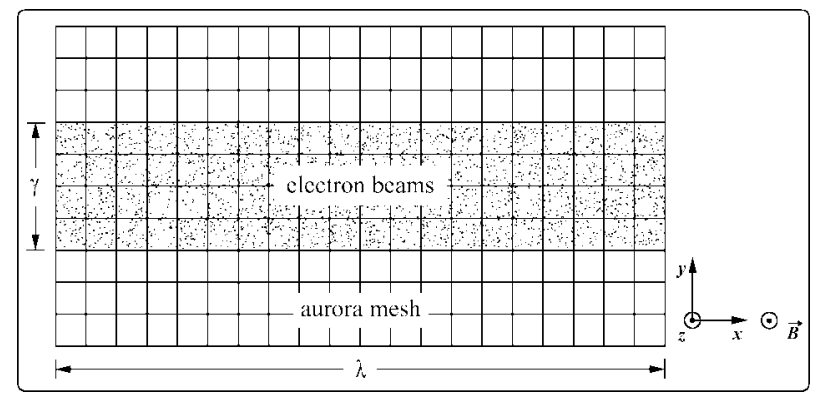

Fig. 5. A cross-section of an auroral stream, model ed by a strip of el ectron beams with periodic and Neuman boundary conditions, along the $x$-axis and $y$-axis, respectively.

and Hockney [1968]. The perpendicular velocities and positions of the electron beams are advanced in time as follows:

(1) from the positions of the electron beams, a charge distribution $(\rho)$ is derived;

(2) from the charge distribution, using Poisson Equation (7), the electrostatic potential is cal culated;

(3) from the potential, the el ectric field ( $\vec{E})$ acting on each el ectron beam is derived using Equation (6);

(4) Newton's law of motion (Equation (2)), using the local el ectron field and the Earth's magnetic field

$(\vec{B})$ is then integrated resulting in a new position and velocity for each electron beam.

The cycle is then repeated at step (1). The aurora dynamics is initiated from incoming sheets of particles at the auroral oval (Figure 1) forming an auroral stream, which is model ed by a strip of incoming el ectron beams with periodic and N euman boundary conditions al ong thex-axis and y-axis respectively. Thestrip in Figure 5 is a small cross-section of part of an auroral oval (Figure 1). As an initial condition, the electron beams are distributed randomly in the interior of this strip of thickness $\gamma$ and length $\lambda$, and have zero perpendicular velocity. Faster initial growth of auroral distortions will occur for larger values of the $\lambda / \gamma$ ratio.

4.1.1 Charge Assignment. In the hybrid Eulerian-Lagrangian formulation, the field and particle quantities are defined on different grids. To obtain the charge density at mesh points from the distribution of particles, we allocate the particle charges based on the $\mathrm{CIC}$ (cloud-in-cell) [Birdsall and Fuss 1969] charge assignment scheme: the charge density $\rho_{i, j}$, at the grid point $G_{(i, j)}$, is given by the sum of weighted charges, $\omega^{k} q_{k}$, of the electron beams $e_{k}$ in the cells surrounding the grid point $G_{(i, j)}$, divided by the cell area:

$$
\rho_{i, j}=\frac{1}{\text { cell area }} \sum_{e_{k} \in \text { cells with vertex }(i, j)} \omega^{k} q_{k},
$$

as indicated in Figure 6(a). However, instead of finding which electron beams contribute to the charge at the grid point $\mathrm{G}_{(i, j)}$, we sweep through each electron beam and distribute its charge with weights $\omega_{i^{*}, j^{*}}^{k} i^{*} \in\{i, i+1\}, j^{*} \in\{j, j+1\}$, to the vertices of the grid cell it bel ongs to, as indicated in Figure $6(b)$. To be consistent with the definition of the continuous charge density (4), the weight $\omega_{\mathrm{i}, \mathrm{j}}^{\mathrm{k}}$ must be given by

$$
\omega_{i, j}^{k}=S_{\epsilon}\left(G_{(i, j)}-P_{k}\right),
$$

where $S_{\epsilon}(\cdot)$ is a modified Dirac delta function with compact support parameterized by $\epsilon$, which has the property that

$$
\lim _{\epsilon \rightarrow 0} S_{\epsilon}(\cdot)=\delta(\cdot)
$$




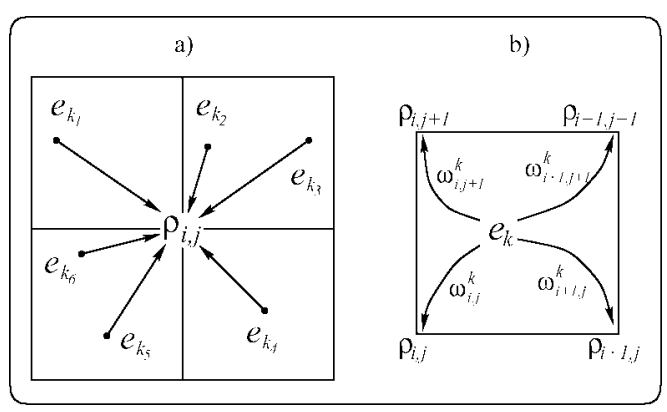

Fig. 6. a) Charge accumulation at grid point $\mathrm{G}_{(\mathrm{i}, \mathrm{j})}$. b) Charge distribution of $\mathrm{e}_{\mathrm{k}}$ to the neighboring grid points.

in the sense of distributions. It can be interpreted physically by ascribing a finite width $(\epsilon)$ to the distribution of charge in each particle. In the $\mathrm{CIC}$ scheme, $\mathrm{S}_{\epsilon}$ is given by the hat-shapefunction [Birdsall and Fuss 1969].

4.1.2 Poisson Equation. Thegradient of the potential function satisfies Equation (7). On an Eulerian grid with mesh size $h$, the partial differential equation can be discretized as

$$
\frac{4 \phi_{i, j}-\phi_{i+1, j}-\phi_{i-1, j}-\phi_{i, j-1}-\phi_{i, j+1}}{h^{2}}=\frac{\rho_{i, j}}{\epsilon_{0}},
$$

which leads to a set of linear equations, denoted by $A \Phi=\rho$, where $\Phi$ is a vector of unknown potentials and $\rho$ is a vector of (scaled) charge densities. The matrix A is large, sparse, symmetric, and positive definite. The complexity of most matrix solvers depends on the size of A. For the simulation of auroral dynamics, a fine grid is required to resolve the layer structure, especially when the formation of surges is simulated. Thus, an efficient linear solver is indispensable.

Direct methods, such as banded Gaussian elimination (GE), can be used to exploit the banded structure of $A$. For matrices of size $n \times n$, the complexity of banded GE is $O\left(n^{2}\right)$, which is still computationally impractical. I terative methods, such as preconditioned conjugate gradient (PCG) with incomplete Cholesky factorization as preconditioner, have been successfully used in computer graphics for fluid simulations [Fedkiw et al. 2001; Foster and Fedkiw 2001]. While the theoretical complexity is reduced to $\mathrm{O}\left(\mathrm{n}^{1.5}\right)$, it is still expensive for the long time simulations presented in this article, which require fine grids as stated above. The FFT method [Stam 1999] can improve the complexity further to $\mathrm{O}\left(\mathrm{n} \log _{2} \mathrm{n}\right)$, but still it is not optimal in the sense given below.

4.1.3 A Practical Multigrid I mplementation. The multigrid method is an optimal scheme in which the convergence rate is independent of the problem size [Trottenber et al. 2001]. Thus, as opposed to the methods previously discussed, it has a linear complexity of $O(n)$, where the complexity constant is known to be small for Poisson equations, typically smaller than 10. It exploits the properties of the linear equations at the PDE level, rather than the matrix structure. It was first proposed by Fedorenko [1961] in the 1960's, and made popular by Brandt [1977] and others for scientific applications. We refer the interested reader to the survey paper by Chan and Wan [2000] on recent developments of linear elliptic multigrid methods. Early attempts in using multigrid methods to solve a multiple scattering problem [Stam 1995] raised the issue of memory due to storage of coarse grid quantities. Here, we introduce a matrix-free implementation which is extremely memory-efficient. We remark that similar multigrid schemes can also be used in other plasma and astrophysical simulations as mentioned in Section 7.

The idea of the multigrid method is to accelerate the convergence of theiterations by solving thePDEs on a sequence of coarse grids. The basic principle is to eliminate high frequency errors by relaxation 


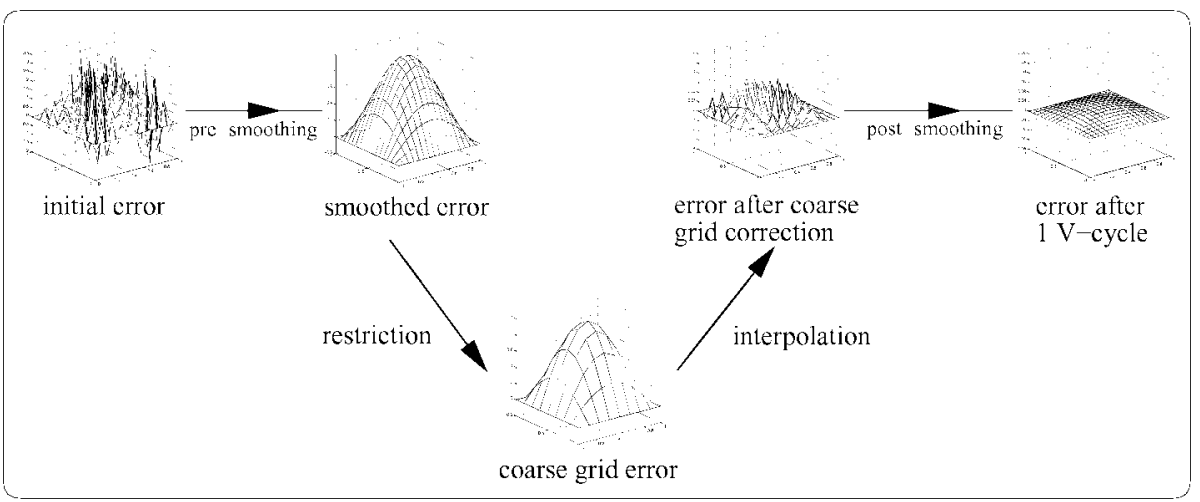

Fig. 7. A multigrid V-cycle: presmoothing, restriction, coarse grid correction, interpolation and post-smoothing.

smoothing, and low frequency errors by coarse grid correction. Let $\Phi^{\mathrm{h}}$ be the current approximate solution on the fine grid with mesh size h, and $\varepsilon^{\mathrm{h}}=\Phi-\Phi^{\mathrm{h}}$ be the error. By the dissipative nature of the Poisson equation, the error in the numerical solution, $\tilde{\Phi}^{h}$, obtained from a few relaxation steps, will become smooth. Thus, one can easily compute an accurate coarse grid approximation by projecting the residual error, $r^{h}=\rho-A \tilde{\Phi}^{h}$, onto the coarse grid with mesh size $H$ and solve the coarse grid error equation

$$
\mathrm{A}^{\mathrm{H}} \varepsilon^{\mathrm{H}}=\mathcal{R}_{\mathrm{h}}^{\mathrm{H}} \mathrm{r}^{\mathrm{h}},
$$

with less computational effort, since there are fewer grid points on the coarse grid. Here $A^{H}$ is the coarse grid matrix and $\mathcal{R}_{\mathrm{h}}^{\mathrm{H}}$ the full weighting restriction operator. Finally, the coarse grid error is then interpolated back to the fine grid to update the fine grid solution

$$
\Phi^{\text {new }}=\tilde{\Phi}^{\mathrm{h}}+\mathcal{P}_{\mathrm{H}}^{\mathrm{h}} \varepsilon^{\mathrm{H}},
$$

where $\mathcal{P}_{H}^{\mathrm{h}}$ is the linear interpolation operator. This process completes one multigrid V-cycle (Figure 7).

We note that only the actions of the coarse grid, interpolation, and restriction operators are needed. Thus, we never form nor store these matrices. Consequently, only 3.5 fine grid vectors are needed in our implementation, which requires even less memory than CG. For the interested reader, an iteration count comparison between our multigrid method and PCG can be found in the original technical report written by the authors [Baranoski et al. 2002].

4.1.4 Velocity and Position U pdate. The electric field $E_{i, j}$ is defined on the Eulerian grid whosevalue is given by numerical differentiation of $\phi_{i, j}$. However, electron beams are located on the Lagrangian grid, which, in general, does not overlap with the Eulerian. Thus, the electric field on an el ectron beam $Q_{k}$, located at $P_{k}$, is obtained by interpolating the electric fields at the nearest four corners, that is, the reverse process of charge distribution as in Figure $6(\mathrm{~b})$. We then update the perpendicular velocities and positions of the electron beams by integrating Equation (2).

\subsection{Trajectories of Electron Beams}

The electron beam's velocity vector, $\vec{v}$, is defined as the overall direction of progression during its descending motion. As the beams travel down, they may suffer several random deflections. Their trajectories are simulated incrementally using the stochastic al gorithm described by Baranoski et al. [2003]. This algorithm takes into account the spatial inhomogeneity of auroral electrons and their initial energy to compute the deflection points and the displacements of an electron beam along the magnetic field lines. 


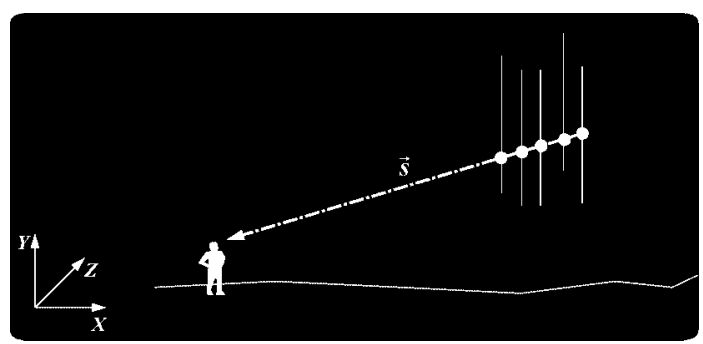

Fig. 8. Sketch showing that the apparent surface brightness of an auroral display is proportional to the number of emissions along the line of sight represented by the vector $\vec{s}$.

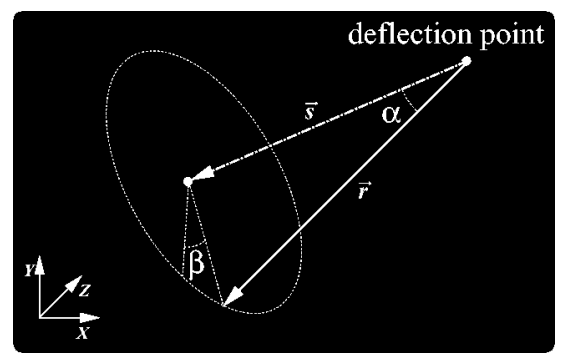

Fig. 9. Diagram showing angular displacements used to distributed emission rays.

Each path is simulated incrementally, using a parametric displacement $u \in[0 . .1]$ such that

$$
u^{\text {new }}=u^{\text {old }}+\left(d_{u} \xi_{1}\right)
$$

where $\xi_{1}$ is an uniformly distributed random number in the interval [0..1], and $d_{u}$ corresponds to a parametric interval (dimensionless) between collisions which is adjusted according to the initial energy of the incoming particles. For instance, an electron with $10 \mathrm{keV}(60000 \mathrm{~km} / \mathrm{s})$ can collide 300 times before becoming indistinguishable from the ambient el ectrons at an altitude of about $100 \mathrm{~km}$ above the ground [Brekke and Egeland 1994]. In this case, we could set $d_{u}=\frac{1}{300}$.

\section{RENDERING ISSUES}

Aurorae are view-dependent phenomena, that is, the apparent surface brightness of an aurora is proportional to the integrated emission-per-unit volume al ong the line of sight (Figure 8). Hence, a forward mapping, or splatting, approach can be used to map auroral emissions to the screen plane [Baranoski et al. 2003; Westover 1991].

Recall that after being hit by an electron, an atmospheric atom becomes excited, and, after a period of time, it may emit a photon. Statistically, the intensity contribution spreads radially around the propagation direction, and follows a Gaussian distribution al ong that dimension [Borovsky et al. 1991; Borovsky and Suszcynsky 1993]. In order to account for this phenomenon, we implemented an al gorithm to perform a distributed forward mapping of auroral emissions. The world coordinates for each deflection point are used to compute the line of sight vector $\vec{s}$. This vector is randomly perturbed through angular displacements $\alpha$ and $\beta$ (Figure9) togeneratea light emission ray $\vec{r}$. Theangle $\alpha$ represents theazimuthal angle around $\overrightarrow{\mathrm{s}}$. The angle $\beta$ represents the polar angle with respect to $\overrightarrow{\mathrm{s}}$, and it corresponds to the exponential glow decay observed in auroral arcs [Borovsky and Suszcynsky 1993]. These angles are 
given by

$$
(\alpha, \beta)=\left(1-\mathrm{e}^{-\frac{\gamma \xi_{2}}{240}}, 2 \pi \xi_{3}\right),
$$

where $\xi_{2}$ and $\xi_{3}$ are uniformly distributed random numbers $\in[0,1]$, and $\gamma$ represents the initial thickness of the auroral stream.

The accuracy and performance of the simulation is dependent on the number, $\mathrm{N}$, of emission rays. Experiments show, however, asymptotic convergence of the results for larger values of $\mathrm{N}$.

The emissions mapped to the screen plane are scaled according to auroral spectral emission and intensity profiles [Baranoski et al. 2003], which correlate with the height of the emission point [Brekke and Egeland 1994]. The conversion of auroral spectral emissions to a color space follows a straightforward CIE XYZ to RGB mapping procedure. We include the strongest auroral spectral emissions in our simulations, namely, 630.0nm (red line), 557.7nm (green line) and 427.8nm (blue line). More wavelengths can be considered at the expense of higher computational time.

The different lifetimes of transition states in auroral emissions-110s for the red line, $0.7 \mathrm{~s}$ for the green line, and $0.001 \mathrm{~s}$ for the blue line-cause the photon emissions to occur in distinct volumes around the principal direction of emission. In order to simulate this distribution of auroral emissions, we convolve the image with a color-dependent Gaussian low-pass filter [Baranoski et al. 2003; Westover 1991].

An auroral display al so exhibits gl obal temporal variations, captured in photographs as blurred forms, due to finite exposure times. For simulating this global blurring effect we perform a convolution using a temporal low-pass filter [Baranoski et al. 2000; Castleman 1996]. The longer the sampled window is in time, the blurrier the results, similar to the effects captured in real photographs with longer exposure times.

The final stage of our rendering pipeline consists of blending the auroral display with a night sky background and a foreground. We use a composite approach in which we initialize the color buffer with the background scene, superimpose the auroral display, and, finally, filter out regions of the image that would be covered by for eground objects.

\section{RESULTS}

In this section, we present examples of simulations of auroral motions and shape changes at different spatio-temporal scales. ${ }^{2}$ Thestochastic nature of auroral displays reduces the viability of a quantitative analysis of their visual simulations. An alternative available is to qual itatively analyze the simulations in comparison with photographs of real auroral displays. However, as mentioned earlier, photographs of an aurora are usually blurred due to low light conditions and significant exposuretimes, and sometimes present background artifacts caused by grain when high speed film is used (Figures 10 and 13). For this reason we accounted for photographic blur in our simulations.

Despitethe blur, features readily recognizable by viewers in real aurorae were preserved, for example, presence of rayed structures and vertical distribution of intensity (Figure 11), as well as their characteristic spatio-temporal variations. This aspect was verified through comparisons with observations of the real phenomena, including the positive feedback from scientists working in the auroral imaging field [Hallinan 2001; Trondsen 2001].

Figures 11, 12 and 14 present still frames from simulation sequences produced using our model. For these sequences, we used a $1024 \times 1024$ mesh for the starting points, and varying values for the vertical displacement of the electron beams. The length and thickness of the initial auroral stream used in these

\footnotetext{
${ }^{2}$ The simulation sequences (quicktime format) described in this article are available at http://www.curumin.uwaterloo. $\mathrm{ca} /{ }^{\sim}$ gvgbaran/dynamics.html.
}

ACM Transactions on Graphics, Vol. 24, No. 1, J anuary 2005. 


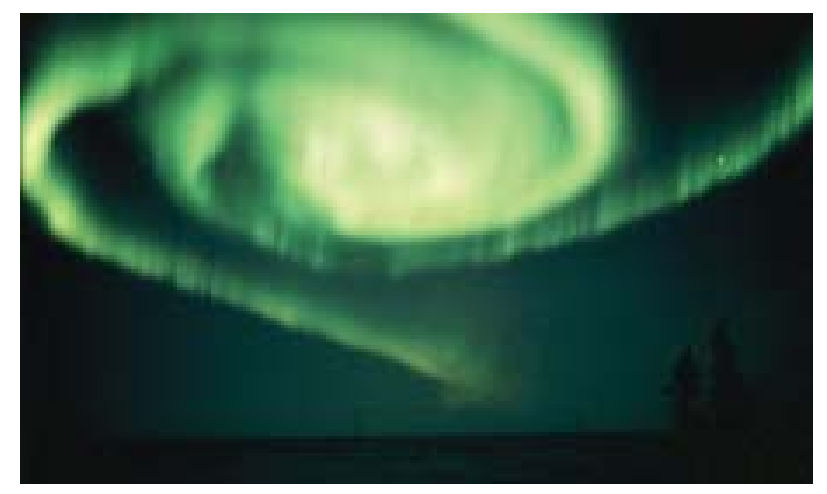

Fig. 10. Photograph of an auroral spiral. (Courtesy of Norbert Rosing.)

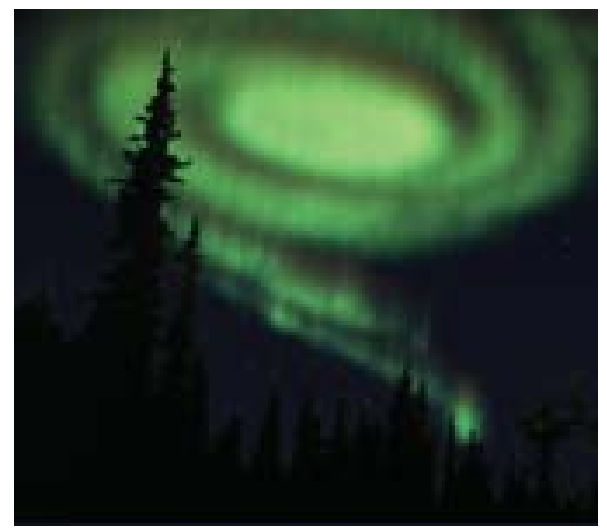

Fig. 11. Simulation of an auroral spiral showing a rayed structure and an arc break up.
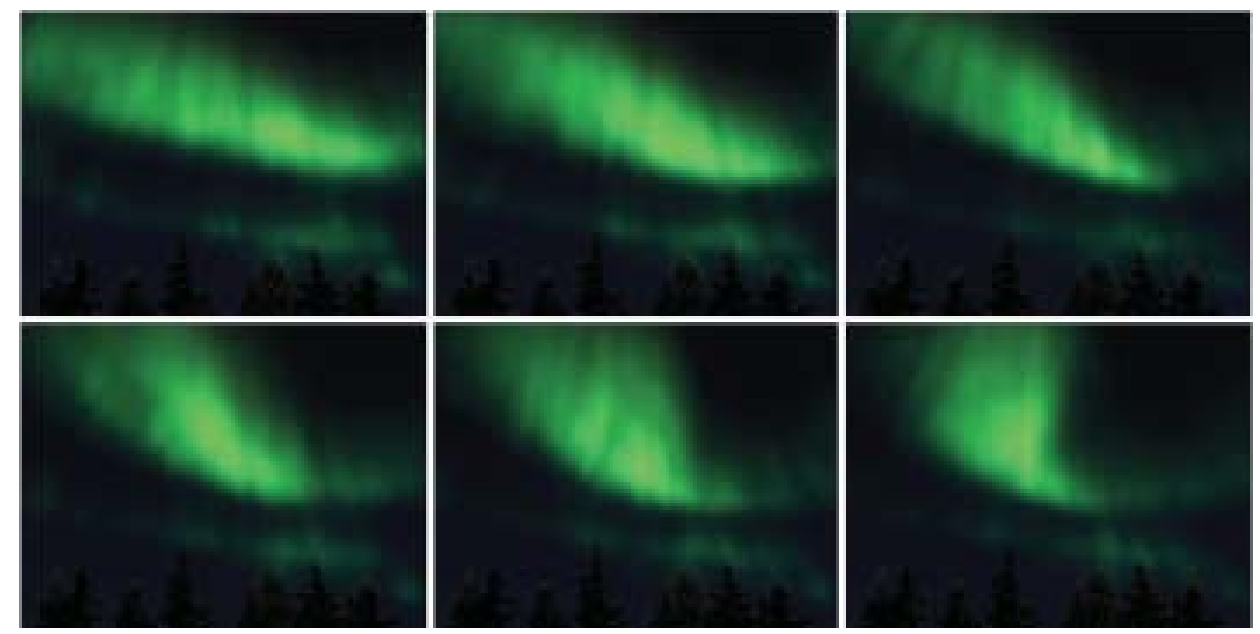

Fig. 12. Frames of a simulation sequence showing the motions and shape changes of an auroral rayed band. 

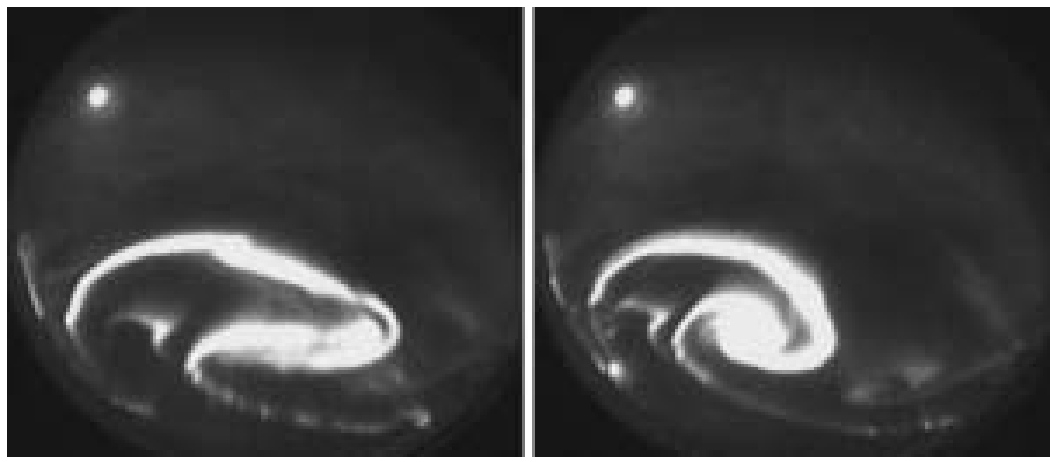

Fig. 13. Black and white all-sky photographs showing different stages of an auroral surge formation. (Courtesy of Kirsti Kauristie.)

simulations were $2000 \mathrm{~km}$, and $9 \mathrm{~km}$, respectively. The computations were performed on a SGI 550, with dual 850Mhz Pentium3 processors.

Figure 12 presents six frames from a simulation sequence showing the motions and shape changes of an auroral rayed band. In this sequence, one can observe the rapid movement of auroral rays, the formation and motion of folds, and a rotational distortion of the auroral band. In this simulation, the number of electron beams was $4 \times 10^{4}$, with a parametric collision interval (Section 4.2) of 0.05. The time spent to compute the starting points and the descending trajectories for each time step was $2.6 \mathrm{~s}$ and 10.8s, respectively. The frames were rendered at a resolution of $320 \times 240$ pixels, and using 10 rays per-emission-point.

Figure 13 presents two all-sky photographs taken during an auroral surge formation in the northern hemisphere. Figure 14 presents three frames from a simulation sequence, illustrating a simulation of such natural phenomena at twice normal speed. In this simulation, the number of el ectrons beams was $10^{4}$, with a parametric collision interval of 0.1 . The time spent to compute the starting points and the descending trajectories for each time step was 1.7s and $6 \mathrm{~s}$, respectively. The frames were rendered at a resolution of $480 \times 360$ pixels, and using 5 rays per-emission-point.

Recently, scientists, using NASA's polar spacecraft, have captured images of the aurorae on film as they were dancing simultaneously around both magnetic poles ${ }^{3}$. This film shows the Aurora Borealis and Aurora Australis expanding and brightening, in parallel, at opposite ends of the Earth, confirming the three-century-old theory that aurorae in the northern and southern hemisphere are nearly mirror images of each other. In the case of auroral spirals, the distinct feature is their opposite sense of rotation. Usually they are counterclockwise in the southern hemisphere when viewed from the ground, and clockwise in the northern hemisphere.

Figure 15 presents six frames from a simulation sequence, illustrating an auroral surge formation in the southern hemisphere. In this sequence, besides the warping process leading to an auroral spiral, we can also notice the formation and motion of folds. In this simulation, there were $10^{4}$ electrons beams, with a parametric collision interval of 0.2 . The time spent to compute the starting points and the descending trajectories for each time step was $1.7 \mathrm{~s}$ and $10.27 \mathrm{~s}$, respectively. The frames were rendered at a resolution of $320 \times 240$ pixels, and using 20 rays per-emission-point.

Using the multigrid algorithm, we were able to reduce substantially the time required in the warping computations. For instance, on the $1024 \times 1024$ auroral mesh used in our simulations, multigrid is 96 times faster than CG. Due to this reduction, the computation of the descending trajectories

\footnotetext{
$\overline{3}$ http://eiger.physics.uiowa.edu/ vis/conjugate_aurora/.
} 

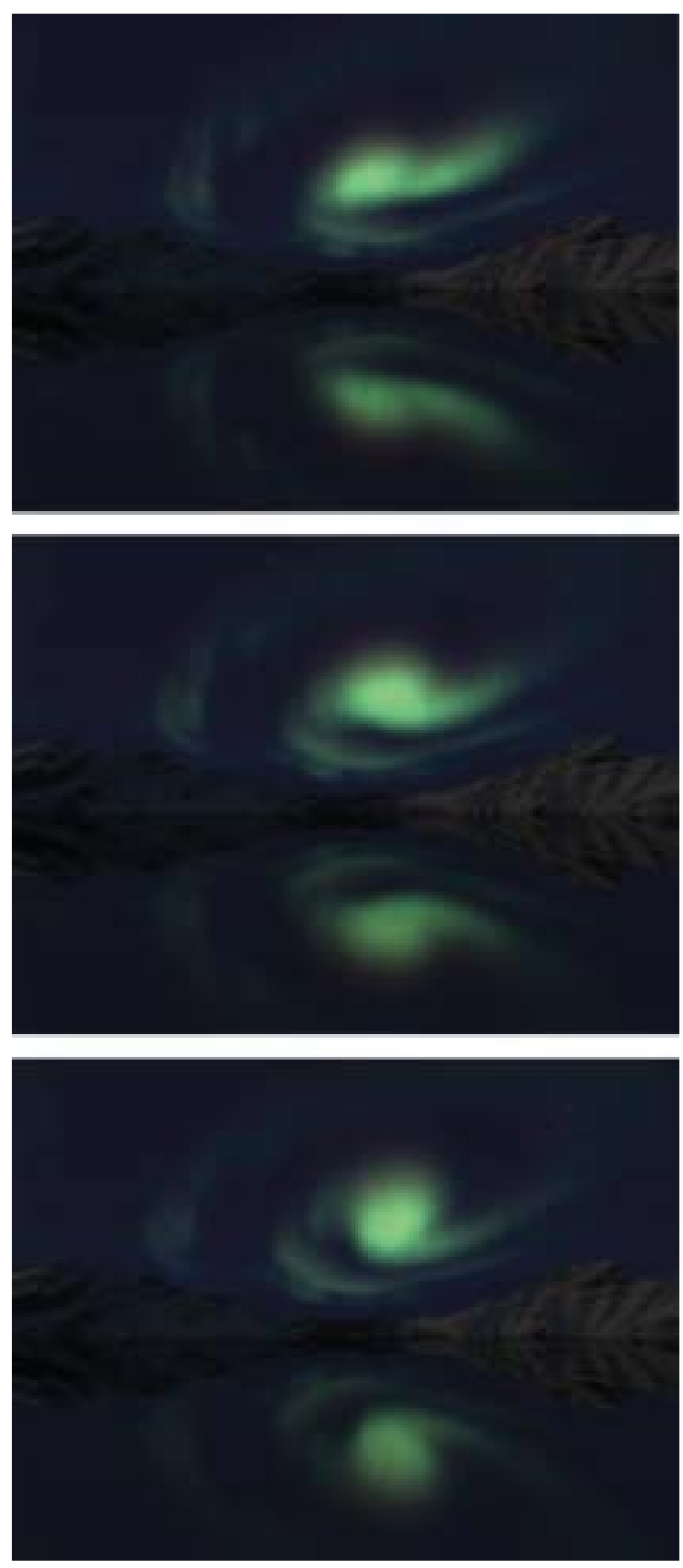

Fig. 14. Frames from a simulation sequence illustrating an auroral surge (spiral) formation in the northern hemisphere. became the bottleneck of our simulation pipeline, with its cost growing linearly with respect to the number of electron beams. A divide-and-conquer strategy can be applied, however, to decrease the total running time of these computations. In other words, one can process $\mathrm{n}$ trajectories separately in several processors. For instance, for $10^{5}$ electron beams, Baranoski and Rokne [2002] obtained a 

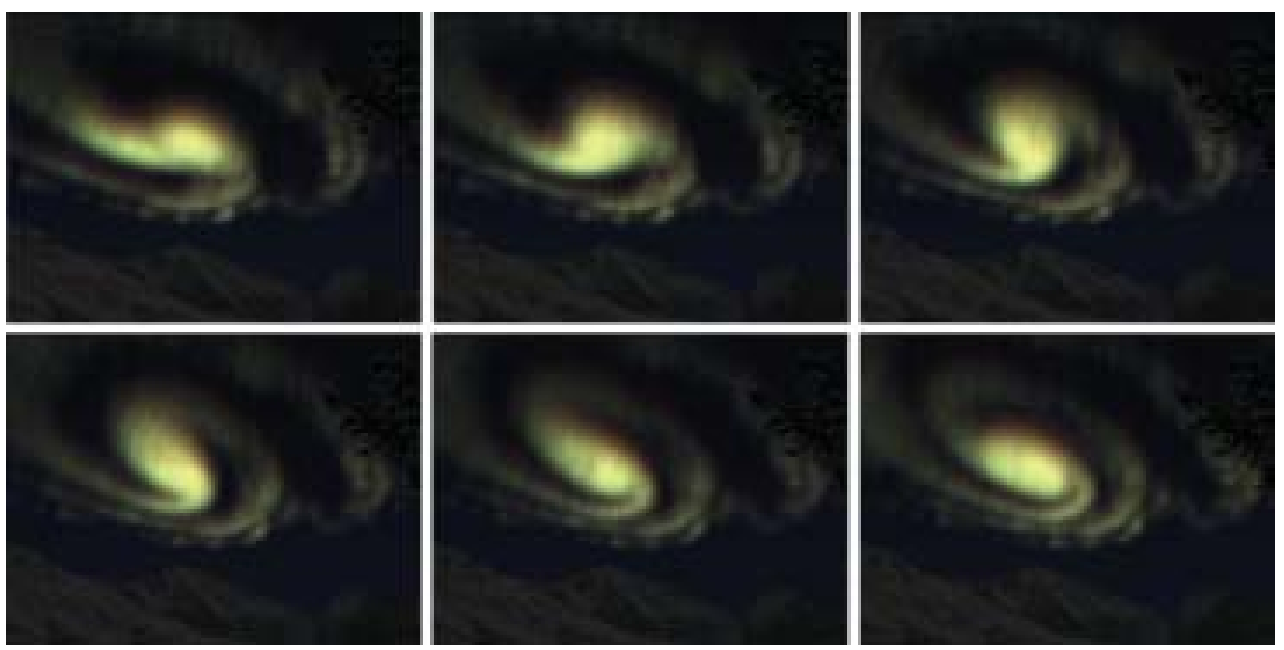

Fig. 15. Frames of a simulation sequence illustrating an auroral surge formation in the southern hemisphere.

speedup of 4.6 using five Compaq Alpha 500M hz PWS processors. Nonetheless, we believe that there is still room for improvement on the parallelization of the algorithms. We intend to continue to work on the parallelization, and to exploit graphics hardware for the real time simulation of moving auroral displays.

\section{DIRECTIONS FOR APPLICATIONS AND FUTURE RESEARCH}

The physical simulation and visual representation of natural phenomena is an evolving area of re search within computer graphics where significant progress has been made. However, the important class of natural processes collectively known as plasma phenomena present significant computational challenges and remain relatively unexplored [Gombosi et al. 2004]. The aurorae are among the most spectacular and most important from a scientific point of view due to the link they provide between solar activities and the Earth. In fact, the aurorae can be thought of as "footprints" of events and energetic process occurring in the Earth's magnetosphere. For this reason, even after 200 years of study, an astonishing amount of basic research is still being done on the aurora and its electromagnetic and electrodynamical properties [Paxton and Meng 1999].

The complexities of numerical simulations in the physical sciences often require visual representations. Complex situations, for example, those found in plasma studies, may be dissected into simpler components to help the understanding of the underlying phenomena and obtain a clear theoretical picture [Tang 2002]. Moreover, animated sequences derived from computer simulations give a picture of a simulated phenomenon through time, illustrating qualitative features of any instabilities that may not be evident from a theoretical point of view. In fact, visualization plays a critical role in going from the raw nonlinear solution of the complex equations governing these phenomena to a simplified model explaining the essential underlying plasma physics as Parker et al. [1995] appropriately stated. This contribution of computer graphics to scientific applications was highlighted by D. Greenberg in the Steven Coons Award Lecture in 1987 [Greenberg 1988]:

If computer graphics is to have a role in improving the future of our civilization, the real value will be in its application to science, engineering and design. 
Viewed in this context, we foresee three main directions for applications and future research associated with the work presented in this article:

-visualization of plasma phenomena,

-visualization of Earth's el ectro-magnetic environment in space, and

- visualization of astrophysical phenomena.

In order to understand plasma phenomena, a combination of extensive data analysis, theory, modeling, laboratory experiments, and in situ (space born) observation is required. Plasma science impacts daily life in many significant ways. In 1995, a panel of scientists at the National Research Council [NRC 1995] recommended that NASA and NSF fund a vigorous observational program related to plasma science, both on Earth and in space. The program should include both in situ and ground-based facilities, properly balanced with programs in theory, modeling, and laboratory experiments (see al so NRC 2003).

In our everyday lives, we encounter plasma in many situations, such as the flash of a lightning bolt, the conducting of gas inside a fluorescent tube or neon sign, the ionization in a rocket exhaust, gas lasers, and lightning discharges as some of the more familiar examples [Chen 1984; Smirnov 2001]. In the neighborhood of Earth, one encounters plasma in the Van Allen radiation belts and the solar winds as soon as one leaves the Earth's atmosphere. Further afar, stellar interiors and atmospheres, as well as gaseous nebulae, are composed of plasma. For example, the Crab Nebula contains a magnetic field and it is a rich source of plasma phenomena. It also contains a visual pulsar. Theories of pulsars picture them as rapidly rotating neutron stars with plasmas emitting synchrotron radiation from the surface [Bittencourt 1986; Chen 1984]. Occasionally, one also encounters an entirely new physical situation which is unlike anything previously observed either in space or in laboratory, and this opens new avenues of research. One example is the dusty plasma of comets and planetary rings that are dominated by the dynamics of charged macroparticles for which gravitational and electromagnetic effects are of comparable importance [NRC 1995].

In the type of particle model used in this article, the computer particle retains much of the identity of the atomic constituents of the material being simulated, yet does not correspond one-to-one to the simulated particles as do atoms to particles in molecular dynamics simulations. The "electron beams" or "superparticles" in these simulations correspond to millions of physical el ectrons. The plasma phenomena mentioned above can also be simulated using this approach. F urthermore, all of the mathematical models to which particle simulation methods are applied can be formulated as a set of particles interacting through fields. The most important field equation is the Poisson's equation, which pertains to all plasma examples listed above, and it is also found in the simulation of clustering of galaxies [Hockney and Eastwood 1988].

Electromagnetism is a fundamental topic [Belcher and Bessette 2001]. It is, however, one of the most difficult subjects for students to master. Belcher and Bessette [2001] addressed this difficulty by creating 3D field line animations used to aid student intuition about el ectromagnetic phenomena occurring in the laboratory. The auroral displays are similarly caused by electromagnetic phenomena, but at a planetary scale, that is, the solar wind impinges on the Earth's magnetosphere, which shields us from this radiation, distorting the field lines of the magnetosphere in the process. Visualization of such phenomena is important not only from a educational point of view [Nylund and Holland 1999], but also from a research point of view since it can be used to support improved predictions of space weather and its effects on systems operating in space as well as on Earth's surface [Williams et al. 1999]. We also note that the magnetic storms responsible for auroral displays can damage satellites, power grids, pipelines, and spacecrafts [Paxton and Meng 1999; Odenwald 2000].

Plasma simulations are rel evant to al most every area of astrophysics, ranging from stellar and interstellar plasmas, to star clusters and galaxies [NRC 1995]. The significant mutual interactions among 
stars and galaxies are provided by Newtonian gravity. The latter is an inverse-square force like the electrostatic force, so it is natural that there should be many similarities between the dynamics of these systems and plasma physics [Binney 1993]. For example, both stellar dynamics and plasma dynamics account for shear (thesimplest model of a stellar disc, which dominates most of galaxies, is the so-called shearing sheet [Binney 1993]) and can be simulated using "superparticles" models. Superparticles in a galaxy model represent millions of real stars. Length and time scales are determined by the dimensions and motion of the spiral arm structures. Collisional effects are made small by smoothing the force of interaction at short range, and by having a large number of superparticles within the range of smoothing [Hockney and Eastwood 1988]. One of the principal applications of such a model is in the study of spiral patterns arising in galaxies [H ockney and Eastwood 1988]. Such patterns are visually similar to the spiral structures arising from our auroral dynamics simulation. This is not a surprise considering that several processes familiar from plasma physics play important roles in structuring stellar systems. Obviously, gravity, unlike the el ectrostatic interaction, is al ways attractive, and this difference between the two systems invariably leaves its imprint [Binney 1993]. Consequently, there are al so differences between the way a given process works in the plasma and stellar-dynamics. We believe, however, that such cross-fertilization is beneficial for both areas of research, and our intent is tofully explorethese parallels.

\section{CONCLUSION}

In this article, we have presented a novel physically-based model for the 3D visual simulation of auroral dynamics. The model employs a complete multigrid PDE simulation of the el ectromagnetic interactions between auroral electrons. It allows an accurate and efficient visualization of auroral phenomena at different spatio-temporal scales. As far as we have been able to ascertain, this article describes the first physically-based visual simulations of these phenomena in the scientific literature, leading to realistic visualization of the shape and the motion of thesefascinating phenomena. Previous auroral simulations have been restricted to the static and KHI free case [Baranoski et al. 2000].

One of the most important research problems facing the computer graphics field since its beginning, is the correct simulation of physical phenomena. As a result, the simulation of natural phenomena such as water, fire, clouds, smoke and so forth. has attracted significant research efforts. These efforts have been concentrated in the less than $1 \%$ of the universe where plasmas are not readily apparent, leaving the remaining $99 \%$ filled with plasma phenomena to be investigated by future researchers. Although scientific and commercial applications have different constraints, we believe that computer graphics researchers working in either area can benefit from efficient and predictive al gorithms for the realistic simulation of plasma phenomena such as the majestic Northern Lights.

\section{ACKNOWLEDGMENTS}

Thanks are due to Trond Trondsen and Thomas Hallinan for their valuable scientific feedback on the simulation of auroral phenomena; Kirsti Kauristie and Norbert Rosing for allowing us to use their auroral photographs; Adriane Baranoski for helping us with the diagrams; Tamer Özsu for his encouragement; NASA Polar Ultraviolet Imager for granting us access to a satellite snapshot of an auroral oval; Gail Chopiak for her logistical assistance; J essica Hodgins and J ohn Hart for their hel pful editorial suggestions; and the anonymous reviewers for their useful insights.

\section{REFERENCES}

Anderson, U. 2001. Time-domain methods for the Maxwell equations. Ph.D. thesis, Department of Numerical Analysis and Computer Science, Royal Institute of Technology, Sweden.

Aruliah, D. ANd Ascher, U. 2002. Multigrid preconditioning for Krylov methods for time-harmonic Maxwell's equations in three dimensions. SIAM J. Scientif. Comput. 24, 2, 702-718. 
Arvo, J. 1995. Analytic methods for simulated light transport. Ph.D. thesis, Yale University.

BarAnoski, G. AND Rokne, J. 2002. Using a HPC system for the simulation of the trajectories of solar wind particles in the ionosphere. In High Performance Computing Systems and Applications, N. Dimopoulos and K. Li, Eds. Kluwer Academic Publishers, Norwell, MA. (Chapter 2), 317-329.

Baranoski, G., Rokne, J., Shirley, P., Trondsen, T., and Bastos, R. 2000. Simulating the aurora borealis. In the 8th Pacific Conference on Computer Graphics and Applications. IEEE Computer Society, Los Alamitos, CA. 2-14.

Baranoski, G., Rokne, J., Shirley, P., Trondsen, T., and Bastos, R. 2003. Simulating the aurora. J. Visualiza. Comput. Animat. 14, 1 (Feb.), 43-59.

Baranoski, G., Wan, J ., Rokne, J. G., And Bell, I. 2002. Simulating the dynamics of the dancing lights. Tech. Rep. CS-2002-16, (April). School of Computer Science, University of Waterloo.

Belcher, J. and Bessette, R. 2001. Using 3D animation in teaching introductory electromagnetism. Comput. Graph. 35, 1 (Feb.), 18-21.

Binney, J. 1993. Gravitational plasmas. In Plasma Physics An Introductory Course, R. Dendy, Ed. Cambridge University Press, Cambridge, UK. 291-318.

BiRdSALL, C. AND FusS, D. 1969. Clouds-in-clouds, clouds-in-cells physics for many-body plasma simulation. J . Comput. Phys. 3, 494-511.

Bittencourt, J. 1986. Fundamentals of Plasma Physics. Pergamon Press, Oxford, UK.

BOROVSKY, J . AND SUSZCYNSKY, D. 1993. Optical measurements of the finestructure of auroral arcs. In Auroral Plasma Dynamics, R. Lysak, Ed. American Geophysical Union, Washington, D.C., 25-30. (Vol. 80 of Geophys. Monogr. Series.)

Borovsky, J., Suszcynsky, D., Buchwald, M., and DeHaven, H. 1991. Measuring the thickness of auroral curtains. Arctic 44, 3, 231-238.

Brandt, A. 1977. Multi-level adaptive solutions to boundary-value problems. Math. Comp. 31, 333-390.

Brekke, A. and Egeland, A. 1994. The Northern Lights, Their Heritage and Science Gröndahl og Dreyers Forlag, AS, Oslo.

BRYANT, D. A. 1999. Electron Accel eration in the Aurora and Beyond. Institute of Physics Publishing, Bristol, UK.

BURTNYK, K. 2000. Anatomy of an aurora. Sky and Telescope 99, 3 (March), 35-40.

Castleman, K. 1996. Digital I mage Processing. Prentice-Hall, New York.

ChAN, T. AND WAN, W. 2000. Robust multigrid methods for elliptic linear systems. J . Comput. Appl. Math. 123, 323-352.

Chen, F. 1984. Introduction to Plasma Physics and Controlled Fusion, 2nd Ed. Plenun Press, New York.

DAVIS, N. 1992. The Aurora Watchers Handbook. University of Alaska Press, Fairbanks.

Davis, T. and Hallinan, T. 1976. Auroral spirals. 1. observations. J. Geophys. Research. 81, 22 (Aug.), $3953-3958$.

Drazin, P. And ReID, W. 1981. Hydrodynamic Stability. Cambridge University Press, Cambridge.

Dupont, D. 2004. Nuclear explosions in orbit. Scientific American, 100-107.

Eather, R. 1980. Majestic Lights. American Geophysical Union, Washington.

EnRIGHt, D., Marschner, S., ANd FEdKIw, R. 2002. Animation and rendering of complex water surfaces. SIGGRAPH Proceedings, Annual Conference Series, 736-744.

Fedkiw, R., Stam, J ., ANd J ensen, H. 2001. Visual simulation of smoke. SIGGRAPH Proceedings, Annual Conference Series, 15-22.

FEdorenko, R. 1961. A relaxation method for solving elliptic difference equations. USSR Comput. Math. Phys. 1, $1092-1096$.

Feldman, B., O'Brien, J ., And Arikan, O. 2003. Animating suspended particle explosions. SIGGRAPH Proceedings, Annual Conference Series, 708-715.

Foster, N. and Fedkiw, R. 2001. Practical animation of fluids. SIGGRAPH Proceedings, Annual Conference Series, $23-30$.

Foster, N. And Metaxas, D. 1997. Modeling the motion of a hot, turbulent gas. SIGGRAPH Proceedings, Annual Conference Series, 181-188.

Golub, G. And LoAn, C. V. 1989. Matrix Computations, Second ed. J ohn Hopkins University Press, Baltimore.

Gombosi, T., Powell, K., Zeeeuw, D., Clauer, C., Hansen, K., Manchester, W., Ridley, A., Rousssev, I., Abd Q. F., Stout, I. S., AND Tотн, G. 2004. Solution-adaptive magnetohydrodynamics for space plasmas: Sun-to-Earth simulations. Computing in Science and Engineering, 14-35.

Greenberg, D. 1988. 1987 Steven A. Coons Award Lecture. Comput. Graph. 22, 1 (Feb.), 7-14.

HaCKBUSCH, W. 1985. Multi-grid Methods and Applications. Springer Verlag, Berlin.

Hallinan, T. 1976. Auroral spirals. 2. theory. J. Geophys. Resear. 81, 22 (Aug.), 3959-3965.

HallinAN, T. 1981. The distribution of vorticity in auroral arcs. In Physics of Auroral Arc Formation, S. Akasofu and J. Kan, Eds. American Geophysicsl Union, Washington, D.C.

Hallinan, T. 2001. Personal communication. Geophysical Institute, University of Alaska. 
Hallinan, T. and Davis, T. 1970. Small-scale auroral distortions. Planet. Space Sci. 18, 1735-1744.

HINZE, J. 1975. Turbulence. McGraw-Hill, New York.

Hockney, R. 1966. Computer experiment of anomalus diffusion. Phys. Fluids 9, 9 (Sept.), 1826-1835.

Hockney, R. AND EASTwOOD, J . 1988. Computer Simulation Using Particles. Institute of Physics, Bristol.

J ensen, H., Durand, F., Stark, M., Premoze, S., Dorsey, J., and Shirley, P. 2001. A physically-based night sky model. SiGgRAPH Proceedings, Annual Conference Series, 399-408.

J ones, A. 1974. Aurora. D. Reidel Publishing Company, Dordrecht, Holland.

Kaj IYA, J. And Herzen, B. V. 1984. Ray tracing volume densities. Comput. Graph. 18, 3 (J uly), 165-174.

KÄRKKÄINEN, K. 2002. On the finite-difference modelling of electromagnetic problems in structured lattices. Ph.D. thesis, Electromagnetics Laboratory, Department of Electrical and Communications Engineering, Helsinki University of Technology, Finland.

Kunimatsu, A., Watanabe, Y., Fuj II, H., Saito, T., Hiwada, K., Takahashı, T., and Ueki, H. $2001 . \quad$ Fast simulation of and rendering techniques for fluid objects. Comput. Graph. For. 20, 3, 57-66.

Levy, R. and Hockney, R. 1968. Computer experiments on low-density crossed-field el ectron beams. Phys. Fluids 11, 4 (April), 766-771.

Ljung, P., Dieckmann, M., Andersson, N., and Ynnerman, A. 2000. Interactive visualization of particle-in-cell simulations. In IEEE Visualization 2000. 469-472.

MIYAZAKI, R., DoBASHI, Y., AND NISHITA, T. 2002. Simulation of cumuliform clouds based on computational fluid dynamics. In Short Presentations of Eurographics 2002, I. Navazo and P. Slusallek, Eds. The Eurographics Association. 405-410.

MyAZAKI, R., Oshida, S., Dobashi, Y., AND NISHITA, T. 2001. A method for modeling clouds based on atmospheric fluid dynamics. In the 9th Pacific Conference on Computer Graphics and Applications. IEEE Computer Society, Los Alamitos, CA. 363-372.

Nadeau, D., Genetti, J., Napear, S., Pailthorpe, B., Emmart, C., Wesselak, E., and Davidson, D. $2001 . \quad$ Visualizing stars and emission nebulas. Comput. Graphics Forum 20, 1 (March), 27-33.

Nguyen, D. Q., Fedkiw, R., And J ensen, H. W. 2002. Physically based modeling and animation of fire. SIGGRAPH Proceedings, Annual ConferenceSeries, 721-728.

NRC. 1995. Plasma Sciencefrom F undamental Research to Technological Applications. National Academy Press, Washington, D.C.

NRC. 2003. The Sun to the Earth and Beyond. National Academy Press, Washington, D.C.

NyLund, S. AND HolLand, D. 1999. Themes and trends in space science data processing and visualization. J ohn Hopkins APL Tech. Digest 20, 4, 533-543.

Odenwald, S. 2000. Solar storms: The silent menace. Sky and Telescope 99, 3 (March), 41-56.

Омноцт, A. 1971. TheOptical Aurora. Springer-Verlag, New York.

Parker, S., Cummings, J ., and Samtaney, R. 1995. Visualization of plasma turbulence. IEe Comput. Graph. Appl. 15, 2, 7-10.

Partamies, N., Freeman, M., and Kauristie, K. 2001. On the winding of auroral spirals: Interhemispheric observations and Hallinan's theory revisited. J. Geophys. Resear. 106 (A12), 28913-28924.

Partamies, N., Kauristie, K., Pulkkinen, T., and Brittnacher, M. 2001. Statistical study of auroral spirals. J. Geophys. Resear. 106, 15415-15428.

Paxton, L. And Meng, C. 1999. Auroral imaging and space-based optical remote sensing. J ohn Hopkins APL Tech. Digest 20, 4, 544-555.

Rasmussen, N., Nguyen, D. Q., Geiger, W., And Fedkiw, R. 2003. Smoke simulation for large scale phenomena. SIGGRAPH Proceedings, Annual Conference Series, 703-707.

Schussman, G., MA, K., Schissel, D., And Evans, T. 2000. Visualizing DIII-D Tokamak magneticfield lines. In IEEE Visualization 2000. 501-504.

Silvester, P. and Ferrari, R. 1990. FiniteElements for Electrical Engineers, 2nd Ed. Cambridge University Press, Cambridge. SmiRnov, B. 2001. Physics of I onized Gases. J ohn Wiley \& Sons, New York.

StAm, J. 1995. Multiple scattering as a diffusion process. In Rendering Techniques'95 (Proceedings of the Sixth Eurographics Rendering Workshop), P. M. Hanrahan and W. Purgathofer, Eds. SIGGRAPH Proceedings, Annual Conference Series, 41-50.

Stam, J. 1999. Stable fluids. SIGGRAPH Proceedings, Annual Conference Series, 121-128.

Stam, J. And Fiume, E. 1993. Turbulent wind fields for gaseous phenomena. SIGGRAPH Proceedings, Annual Conference Series, 369-376.

TAflove, A. 2000. Computational Electrodynamics: Thefinitedifferencetimedomain method, 2nd Ed. Artech House, Boston, MA.

ACM Transactions on Graphics, Vol. 24, No. 1, J anuary 2005. 
TAjımA, T. 1989. Computational Plasma Physics: With Applications to Fusion and Astrophysics. Addison-Wesley, Redwood City, CA.

TANG, W. 2002. Advanced computations in plasma physics. Phys. Plasmas 9, 5, 1856-1872.

TAYLOR, K. 2001. Auroras earth's grand show of lights. National Geographic, 48-63.

Trondsen, T. 1998. High spatial and temporal resolution auroral imaging. Ph.D. thesis, Department of Physics, Faculty of Science, University of Tromsø, Norway.

Trondsen, T. 2001. Personal communication. Institute for Space Research, The University of Calgary.

Trottenber, U., Oosterlee, C., Schüller, A., Brandt, A., Oswald, P., and Stüben, K. $2001 . \quad$ Multigrid. Academic Press, New York.

Upson, C., Barr, A., Reeves, B., Wolff, R., and Wolfram, S. 1987. The physical simulation and visual representation of natural phenomena. Comput. Graph. 21, 4, 355-356.

VolAKIS, J. AND KEMPEL, L. 1995. Electromagnetics: Computational methods and considerations. IEEE Computat. Sci. Engineer. 2, 1, 42-57.

Weimer, H. and Warren, J. 1999. Subdivision schemes for fluid flow. SIGGRAPH Proceedings, Annual Conference Series, 111-120.

Westover, L. 1991. Splatting: A parallel, feed-forward volume rendering algorithm. Ph.D. thesis, Department of Computer Science, University of North Carolina at Chapel Hill.

Williams, D., Mauk, B., Mitchell, D., Roelof, E., and Zanetti, L. 1999. Radiation belts and beyond. J ohn Hopkins APL Tech. Digest 20, 4, 544-555.

WitKIn, A. And Kass, M. 1991. Reaction-diffusion textures. Comput. Graph. (SIGGRAPH Proceedings) 25, 4, $299-308$.

Yoshizawa, A. 1998. Hydrodynamic and Magnetohydrodynamic Turbulent Flows: Modelling and Statistical Theory. Kluver, Dordrecht.

Yoshizawa, A., Iтон, S., Iтон, K., And Yokol, N. 2001. Turbulence theories and modelling of fluids and plasma. Plasma Phys. and Controlled Fusion 43, 1-144.

Received May 2002; accepted October 2004 